\title{
MicroRNA-744 promotes proliferation of osteosarcoma cells by targeting PTEN
}

\author{
WEI YU $^{1 *}$, PENG-BO CHEN ${ }^{2 *}$, FAN-CHENG CHEN ${ }^{3}$, SHENG-LONG DING $^{4}$ and XIAO-YUN PAN ${ }^{1}$ \\ ${ }^{1}$ Department of Orthopedic Surgery, The Second Affiliated Hospital of Wenzhou Medical University, \\ Wenzhou, Zhejiang 325000; ${ }^{2}$ Department of Orthopedic Surgery, Xinhua Hospital, \\ Shanghai Jiaotong University School of Medicine, Shanghai 200092; ${ }^{3}$ Department of Orthopedic Surgery, \\ Zhongshan Hospital, Fudan University, Shanghai 200433; ${ }^{4}$ Department of Orthopedics, \\ Qingpu Branch of Zhongshan Hospital, Fudan University, Shanghai 201700, P.R. China
}

Received October 27, 2019; Accepted February 25, 2020

DOI: $10.3892 / \mathrm{mmr} .2020 .11030$

\begin{abstract}
MicroRNAs (miRNAs/miRs) are non-coding RNAs that regulate protein synthesis by targeting mRNAs for translational repression or degradation. Previous studies have reported that aberrant expression of miR-744 may be involved in human osteosarcoma; however, the underlying mechanisms remain elusive. In the present study, the expression levels of miR-744 and its downstream signals were determined by reverse transcription-quantitative PCR and western blotting. Cell proliferation was assessed using the bromodeoxyuridine assay, and the target of miR-744 was investigated using a dual-luciferase activity assay. The present study identified a significant upregulation of miR-744 in osteosarcoma tissues compared with adjacent non-tumor tissues. Furthermore, it was demonstrated that ectopic overexpression of miR-744 induced by a miR-744 precursor significantly enhanced proliferation of the osteosarcoma cell line MG63, whereas opposite results were observed following suppression of miR-744 with its inhibitor. Moreover, as a unique anti-oncogene, PTEN was identified as a direct target of miR-744. It was confirmed that miR-744 downregulated PTEN expression in MG63 cells by targeting the PTEN 3'untranslated region, and that the downstream AKT signal was also regulated by miR-744. Collectively, the present results suggested that miR-744 promoted proliferation of human osteosarcoma cells by directly regulating the PTEN/AKT signaling pathway.
\end{abstract}

Correspondence to: Dr Xiao-Yun Pan, Department of Orthopedic Surgery, The Second Affiliated Hospital of Wenzhou Medical University, 109 Xue Yuan Xi Road, Wenzhou, Zhejiang 325000, P.R. China

E-mail:wzfeygk@163.com

${ }^{*}$ Contributed equally

Key words: osteosarcoma, microRNA, PTEN, cell proliferation

\section{Introduction}

MicroRNAs (miRNAs/miRs) are a class of small, non-coding single-stranded RNAs that either repress translation or degrade mRNA by base pairing to the $3^{\prime}$ untranslated region (3'UTR), thus contributing to inhibition of target gene expression $(1,2)$. miRNAs are involved in various physiological and pathological processes, including carcinogenesis, cell proliferation and differentiation (2). Moreover, it has been reported that miRNA alterations are associated with the initiation, progression and metastasis of human cancer $(3,4)$. Osteosarcoma is a common primary malignant bone tumor mainly occurring in childhood and adolescence, which has a high mortality rate, with a 5-year survival rate of $<60 \%$, due to robust invasion and metastasis (5). While treatment for osteosarcoma has progressed, the prognosis of osteosarcoma remains poor, with a relatively unchanged 5-year survival rate of $65 \%$ over the last 30 years (6). Furthermore, the pathogenesis of osteosarcoma is not fully understood.

Abnormal miRNA expression may be involved in the occurrence and development of osteosarcoma. It has been reported that miR-144 suppressed tumor growth and metastasis in osteosarcoma via dual-suppression of the Ras homolog family member A and Rho-associated coiled-coil-containing protein kinase 1 signaling pathways (7). However, miR-181a and miR-199b-5p have been shown to promote cell proliferation, invasion and metastasis in osteosarcoma $(8,9)$. Furthermore, a recent study identified the involvement of miR-744 in osteosarcoma and chemotherapy resistance (10). Sun et al (11) revealed that miR-744 promoted osteosarcoma development and progression by suppressing large tumor suppressor kinase 2 (LATS2) signaling. However, due to the limited number of studies examining miR-744 in osteosarcoma, the role of miR-744 and its underlying molecular mechanisms in the pathogenesis of osteosarcoma are not fully understood. PTEN, a unique tumor suppressor gene, has been identified to be a targeted gene of miR-744 in laryngeal squamous cell carcinoma (12), and has also been shown to be associated with the growth and metastasis of various malignant tumors (13). Therefore, the aim of the present study was to investigate whether PTEN is a direct target of miR-744 in the regulation of osteosarcoma cell proliferation. 


\section{Materials and methods}

Cell culture and tissue samples. The MG63, U2OS and SaOS2 human osteosarcoma cell lines were obtained from The Cell Bank of Type Culture Collection of the Chinese Academy of Sciences. The hFOB1.19 human fetal osteoblastic cell line was obtained from the Institutes for Biological Sciences, Chinese Academy of Sciences. Human osteosarcoma cell lines were cultured in DMEM (Gibco; Thermo Fisher Scientific, Inc.), and the human fetal osteoblastic cell line was cultured in DMEM-F12 medium (Gibco; Thermo Fisher Scientific, Inc.). The aforementioned media were supplemented with $10 \%$ FBS (Gibco; Thermo Fisher Scientific, Inc.) and $1 \%$ penicillin/streptomycin. Cells were maintained and subcultured at $37^{\circ} \mathrm{C}$ in a humidified incubator containing $5 \% \mathrm{CO}_{2}$. Cells were pre-treated with $0.1 \%$ DMSO (control) or $20 \mu \mathrm{M} \mathrm{LY} 294002$ (dissolved in 0.1\% DMSO; Sigma-Aldrich; Merck KGaA) for $8 \mathrm{~h}$ at $37^{\circ} \mathrm{C}$.

In total, 25 osteosarcoma tissue samples and corresponding adjacent non-tumor tissues $(>2 \mathrm{~cm}$ from the tumor margin) were obtained from the Department of Orthopedic Surgery, The Second Affiliated Hospital of Wenzhou Medical University. Tissue samples were collected from patients undergoing complete resection surgery between July 2015 and June 2019. Informed consent was obtained from the patients (age, $\geq 18$ years) or their parents (patients aged $<18$ years), and all patients had not received any radiation therapy or chemotherapy prior to surgery. The samples were obtained from 15 male patients and 10 female patients (age, 23.6 \pm 8.6 years; age range, 12-43 years) with an average tumor size of $8.7 \pm 3.5 \mathrm{~cm}$ (tumor size range, $3.8-16.1 \mathrm{~cm}$ ). With regards to tumor subtype, 21 were high-grade osteosarcoma, one was intermediate-grade osteosarcoma and three were low-grade osteosarcoma. WHO classification was used to identify the osteosarcoma subtypes based on the characteristics of tumor tissue observed under the microscope (14). This study was approved by the Ethics Committee of The Second Affiliated Hospital, Wenzhou Medical University.

Analysis of miRNA expression using reverse transcription-quantitative PCR (RT-qPCR). Total RNA was extracted from tissue samples and cell lines using TRIzol ${ }^{\circledR}$ reagent (Invitrogen; Thermo Fisher Scientific, Inc.), according to the manufacturer's protocol. RNA was reverse transcribed into cDNA using stem-loop primers and the TaqMan miRNA RT kit (Applied Biosystems; Thermo Fisher Scientific, Inc.). The temperature and duration conditions of RT were as follows: $37^{\circ} \mathrm{C}$ for $15 \mathrm{~min}$ and $85^{\circ} \mathrm{C}$ for $5 \mathrm{sec}$. The specific primer sequences were as follows: miR-744, forward 5'-ACA CTCCAGCTGGGTGCGGGGCTAGGGCTAAC-3', reverse 5'-CTCAACTGGTGTCGTGGA-3'; and U6 small nuclear RNA, forward 5'-CTCGCTTCGGCAGCACA-3' and reverse 5'-AACGCTTCACGAATTTGCGT-3'. miR-744 expression was detected using a TaqMan human miRNA assay (Applied Biosystems; Thermo Fisher Scientific, Inc.) according to the manufacturer's protocol. qPCR was performed using an Applied Biosystems 7500 Sequence Detection system (Applied Biosystems; Thermo Fisher Scientific, Inc.) with U6 small nuclear RNA as the internal control. The following thermocycling conditions were used: Initial denaturation at $95^{\circ} \mathrm{C}$ for $5 \mathrm{~min}$; followed by 40 cycles at $95^{\circ} \mathrm{C}$ for $15 \mathrm{sec}$, and primer annealing and extension at $60^{\circ} \mathrm{C}$ for $30 \mathrm{sec}$; and final extension for $1 \mathrm{~min}$ at $72^{\circ} \mathrm{C}$. The relative expression of miR-744 was calculated using the $2^{-\Delta \Delta \mathrm{Cq}}$ method (15).

Cell transfection. miR-744 precursor (5'-UGCGGGGCUAGG GCUAACAGCA-3'; final concentration, $100 \mathrm{nM}$ ), miR-744 inhibitor (anti-miR-744; 5'-UGCUGUUAGCCCUAGCCC CGCA-3'; final concentration, $100 \mathrm{nM}$ ) and their corresponding miR negative controls (miR-control; 5'-UUCUCC GAACGUGUCACGUTT-3'; final concentration, $100 \mathrm{nM}$; and anti-miR-control; 5'-CAGUACUUUUGUGUAGUA CAA-3'; final concentration, $100 \mathrm{nM}$ ) were purchased from Shanghai GenePharma Co., Ltd. At 60-70\% confluency, MG63 osteosarcoma cells were transfected for $6 \mathrm{~h}$ at $37^{\circ} \mathrm{C}$ using Lipofectamine ${ }^{\circledR} 2000$ reagent (Invitrogen; Thermo Fisher Scientific, Inc.). Cells were then transferred to complete medium and incubated at $37^{\circ} \mathrm{C}$ for $48 \mathrm{~h}$ before harvesting. Transfection efficiency was subsequently evaluated by RT-qPCR at $48 \mathrm{~h}$ after transfection. Subsequent experiments were performed at $24 \mathrm{~h}$ post-transfection.

Bromodeoxyuridine (BrdU) assay. Cell proliferation was assessed using an ELISA BrdU kit (cat. no. Roche-11647229001; Sigma-Aldrich; Merck KGaA). Following successful transfection, cells were seeded in 96-well plates at a density of $2 \times 10^{3}$ cells/well. The BrdU assay was subsequently performed according to the manufacturer's protocol. Absorbance was measured at a wavelength of $450 \mathrm{~nm}$ using an enzyme immunoassay analyzer (Bio-Rad Laboratories, Inc.). To determine the cell number, cells collected, centrifuged at $150 \mathrm{x} g$ for $5 \mathrm{~min}$ at room temperature, resuspended and counted using a light microscope (magnification, x400) with a Neubauer counting chamber. The experiment was repeated $\geq 3$ times.

Western blot analysis. To isolate proteins, cells or tissues were harvested and lysed with ice-cold RIPA lysis buffer (Beyotime Institute of Biotechnology). The lysates were centrifuged at $20,000 \mathrm{x} \mathrm{g}$ for $15 \mathrm{~min}$ at $4^{\circ} \mathrm{C}$. Proteins in the supernatants were then collected and quantified using a bicinchoninic acid protein assay kit (Beyotime Institute of Biotechnology). Equal amounts of protein (40 $\mu \mathrm{g} /$ lane) were separated by SDS-PAGE on $10 \%$ gels, and then transferred to PVDF membranes (Bio-Rad Laboratories, Inc.). After blocking with 5\% non-fat milk in TBS-Tween (TBST; $50 \mathrm{mM}$ Tris-HCl; pH 7.5; $150 \mathrm{mM}$ $\mathrm{NaCl} ; 0.05 \%(\mathrm{v} / \mathrm{v})$ Tween 20 ) for $2 \mathrm{~h}$ at room temperature, membranes were incubated with specific primary antibodies overnight at $4^{\circ} \mathrm{C}$, including anti-phosphorylated (p)-PTEN (cat. no. 9554; 1:500), anti-PTEN (cat. no. 9552; 1:500), anti-p-AKT (cat. no. 4060; 1:1,000), anti-AKT (cat. no. 9272; 1:1,000) and anti-GAPDH (cat. no. 8884; 1:1,000), which were purchased from Cell Signaling Technology, Inc. After washing three times with TBST, membranes were incubated with horseradish peroxidase-linked rabbit anti-mouse secondary antibody (cat. no. A0216; 1:2,000; Beyotime Institute of Biotechnology) at room temperature for $2 \mathrm{~h}$. The protein signals were detected using an enhanced chemiluminescence system western blotting kit (GE Healthcare Bio-Sciences), according to the manufacturer's protocol. The density of protein bands was semi-quantified by ImageJ software (version 1.6; National Institutes of Health) and the value was normalized to that of GAPDH. 

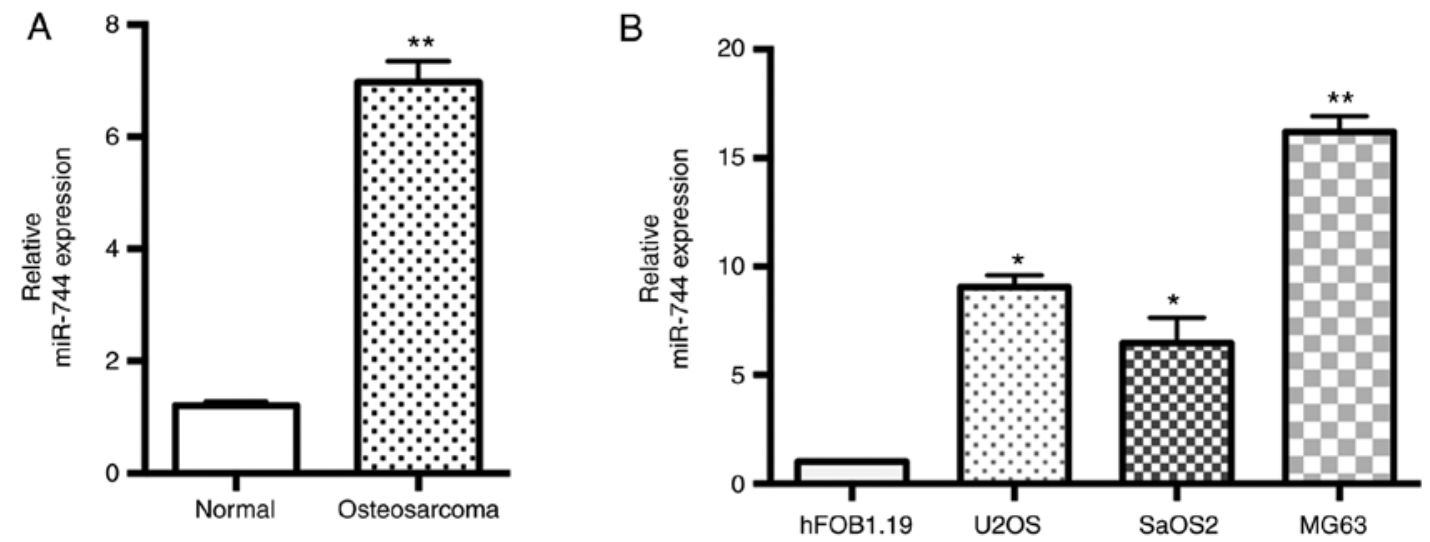

Figure 1. miR-744 expression is increased in osteosarcoma tissues and cell lines. (A) miR-744 expression was detected in 25 pairs of human osteosarcoma tissues and matched adjacent non-malignant tissues (Normal) using TaqMan RT-qPCR assay; U6 was used as the internal control. ${ }^{* *} \mathrm{P}<0.01 \mathrm{vs.} \mathrm{Normal.}$ (B) miR-744 expression was detected in three osteosarcoma cell lines (U2OS, SaOS2 and MG63) and a human osteoblastic cell line (hFOB1.19) by RT-qPCR. ${ }^{*} \mathrm{P}<0.05,{ }^{* *} \mathrm{P}<0.01$ vs. hFOB1.19. miR-744, microRNA-744; RT-qPCR, reverse transcription-quantitative PCR.

Luciferase reporter assay. TargetScan (version 7.2; www. targetscan.org/vert_72) and miRanda (version 2010; www. microrna.org/microrna/home.do) were used to predict the target sites for miR-744, and subsequently identified binding sites between PTEN and miR-744. The predicted wild-type and mutant 3'UTRs of PTEN containing the binding sites to hsa-miR-744 were cloned by PCR amplification. Genomic DNA of MG63 cell was used as the template. PCR was performed using the Takara ${ }^{\mathrm{TM}}$ LA Taq DNA polymerase kit (Takara Bio, Inc.) with the following thermocycling conditions: Denaturation at $95^{\circ} \mathrm{C}$ for $5 \mathrm{~min}$; followed by 30 cycles at $95^{\circ} \mathrm{C}$ for $15 \mathrm{sec}$; primer annealing and extension at $72^{\circ} \mathrm{C}$ for $30 \mathrm{sec}$; followed by 15 cycles at $95^{\circ} \mathrm{C}$ for $15 \mathrm{sec}, 53^{\circ} \mathrm{C}$ for $30 \mathrm{sec}, 72^{\circ} \mathrm{C}$ for $30 \mathrm{sec}$; and a final extension step of $10 \mathrm{~min}$ at $72^{\circ} \mathrm{C}$. Subsequently, genes were inserted into the pMIR-REPORT luciferase vector (Applied Biosystems; Thermo Fisher Scientific, Inc.) at the SacI and HindIII sites. The following primers were used to amplify specific fragments: Wild-type PTEN forward, 5'-CCACAT CCTACCCCTTTGCACTGGCAACAGATAAGTTTGCAG TTGGCTAA-3' and reverse, 5'-AGCTTTAGCCAACTGCAA ACTTATCTGTTGCCAGTGCAAAGGGGTAGGATGTGG ACT-3'; and mutant PTEN forward, 5'-CCACATCCTACCCCT TTGCACCTATCGTCGATAAGTTTGCAGTTGGCTAA-3' and reverse, 5'-AGCTTTAGCCAACTGCAAACTTATCGAC GATAGGTGCAAAGGGGTAGGATGTGGAGCT-3'. For the reporter assay, all cells were seeded ( $1 \times 10^{5}$ cells/well) in 24-well plates. At $70 \%$ confluency, pMIR-REPORT vector $(200 \mathrm{ng})$ containing the wild-type or mutant 3'UTR of PTEN, along with miR-744 precursor (200 ng) or anti-miR-744 (200 ng) were co-transfected into the cells using Lipofectamine ${ }^{\circledR} 2000$ (Invitrogen; Thermo Fisher Scientific, Inc.), according to the manufacturer's protocol. After transfection at $37^{\circ} \mathrm{C}$ for $36 \mathrm{~h}$, cells were lysed using $0.25 \%$ trypsin-EDTA and the luciferase activity was determined using a dual-luciferase reporter assay system (Promega Corporation). Relative firefly luciferase activity was normalized to Renilla luciferase activity.

Statistical analysis. Experiments were repeated $\geq 3$ times, and data are presented as the mean \pm standard error of the mean. Differences between groups were analyzed using a paired Student's t-test or one-way ANOVA followed by a Tukey's multiple comparison post hoc test. Statistical analyses were performed using GraphPad Prism 5.0 software (GraphPad Software, Inc.) and SPSS software version 18 (IBM Corp.). $\mathrm{P}<0.05$ was considered to indicate a statistically significant difference.

\section{Results}

miR-744 expression is upregulated in osteosarcoma tissues and cells. miR-744 expression in the 25 pairs of human osteosarcoma tissues and adjacent non-malignant tissues was assessed using the TaqMan RT-qPCR assay. It was revealed that the expression levels of miR-744 were significantly increased in osteosarcoma tissues compared with the adjacent non-malignant tissues (Fig. 1A). Moreover, osteosarcoma cell lines (U2OS, SaOS2 and MG63) expressed higher levels of miR-744 compared with the human fetal osteoblastic cell line hFOB1.19 (Fig. 1B). The MG63 cell line was selected for further studies as it exhibited the highest expression of miR-744 among the three osteosarcoma cell lines. Based on the overexpression of miR-744 in osteosarcoma tissues and cells, it was hypothesized that miR-744 may act as a functional miRNA in osteosarcoma, particularly in the MG63 cell line.

miR-744 enhances proliferation of osteosarcoma cells. In order to investigate whether miR-744 was involved in osteosarcoma carcinogenesis, miR-744 precursor and anti-miR-744 were transiently transfected into MG63 cells. The effects of miR-744 on osteosarcoma cell proliferation were subsequently assessed by BrdU assays. After $48 \mathrm{~h}$, as determined by RT-qPCR, miR-744 was significantly overexpressed or knocked down by miR-744 precursor and anti-miR-744, respectively (Fig. 2A). Furthermore, BrdU assay results demonstrated that miR-744 overexpression significantly enhanced the proliferation of MG63 cells. However, knockdown of miR-744 significantly inhibited the proliferation of MG63 cells (Fig. 2B). Meanwhile, cell counting experiments indicated that miR-744 overexpression or knockdown resulted in significance alterations to the cell numbers within 3 days (Fig. 2C). The miR-744 overexpression or knockdown-mediated effects on cell numbers were more obvious with incubations $>3$ days (data not shown). 

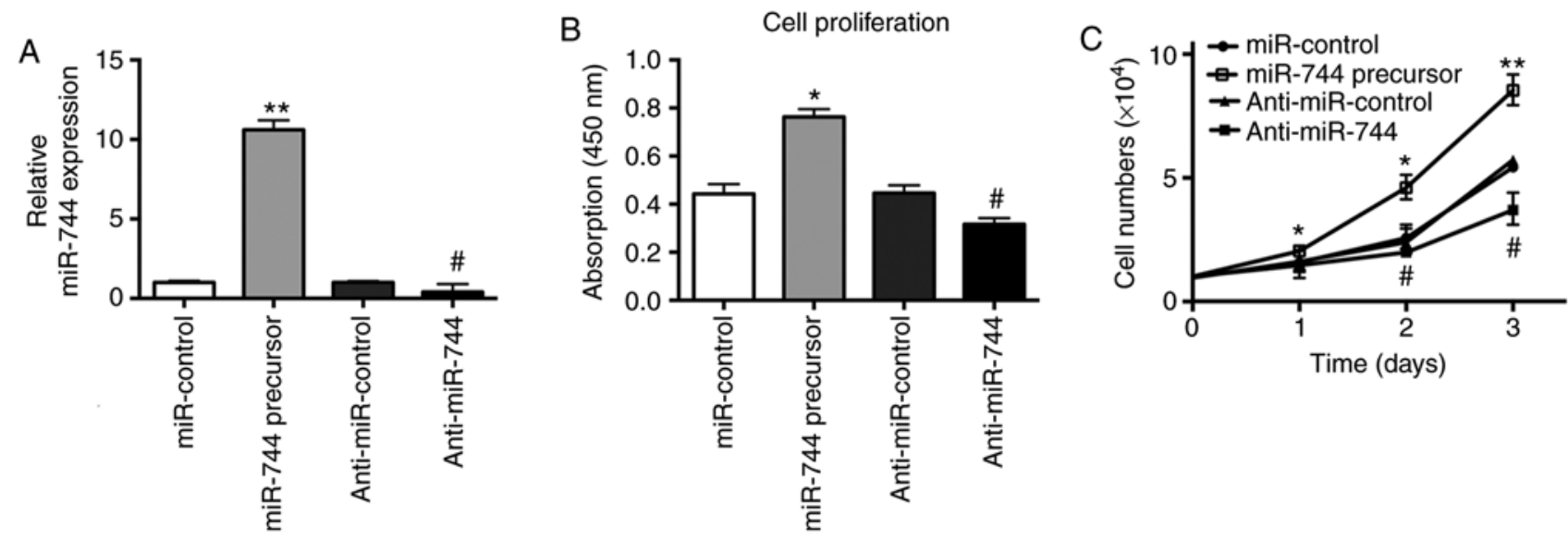

Figure 2. Effects of miR-744 on the proliferation of osteosarcoma cells. (A) Successful transfection of miR-744 precursor or anti-miR-744 in MG63 cells was assessed by reverse transcription-quantitative PCR. (B) Effects of miR-744 on cell proliferation were determined by bromodeoxyuridine assay following transfection for $24 \mathrm{~h}$. (C) Proliferation curve of MG63 cells following transfection. ${ }^{*} \mathrm{P}<0.05,{ }^{* *} \mathrm{P}<0.01$ vs. miR-control; " $\mathrm{P}<0.05$ vs. Anti-miR-control. miR, microRNA.

A

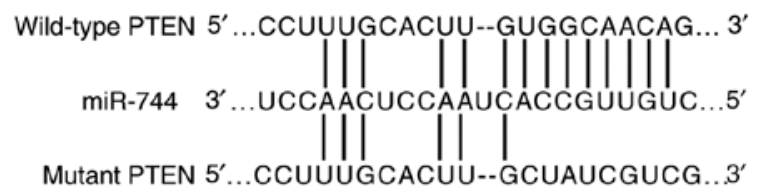

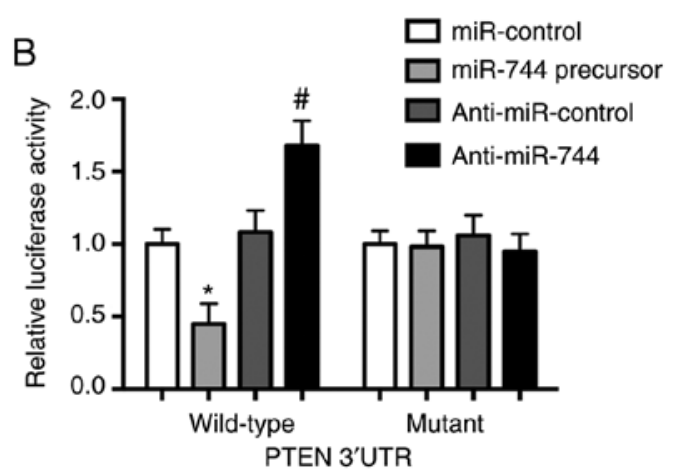

C

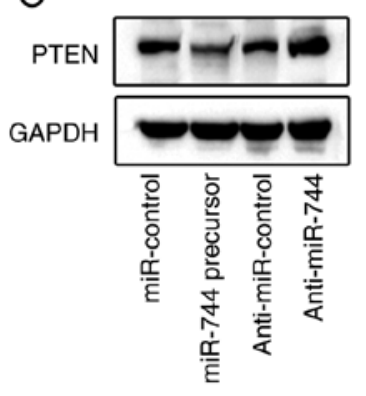

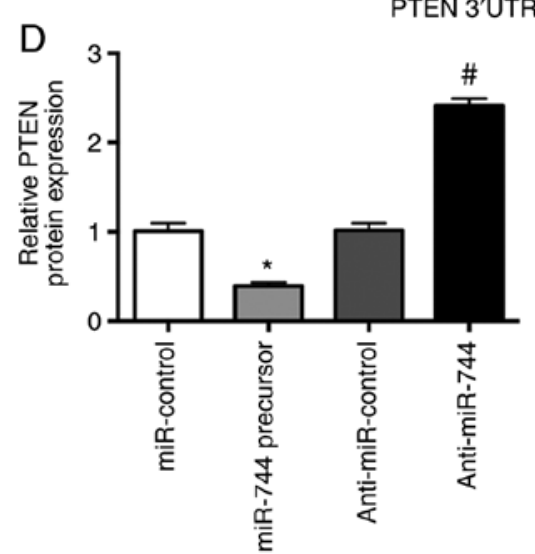

Figure 3. miR-744 negatively regulates PTEN expression in MG63 cells. (A) Predicted sequences of miR-744 binding sites in the 3'UTR of human PTEN gene. Potential binding sites are highlighted. (B) Relative luciferase activity was detected in MG63 cells following co-transfection with wild-type or mutant PTEN reporter plasmids, and miR-744 precursor or anti-miR-744. (C) Representative images and (D) semi-quantitative analysis of PTEN protein expression in MG63 cells were obtained by western blotting, following transfection with miR-744 precursor, anti-miR-744 or negative controls for $24 \mathrm{~h}$. GAPDH was used as an internal control. "P<0.05 vs. miR-control; "P<0.05 vs. Anti-miR-control. 3'UTR, 3' untranslated region; miR, microRNA.

PTEN is a direct target of miR-744 in osteosarcoma cells. Bioinformatics analysis using TargetScan 7.2 and miRanda suggested various potential target genes for miR-744. Of these potential candidates, PTEN, which is a tumor suppressor gene (13), harbored potential miR-744 binding sites within its 3'UTR (Fig. 3A). To further assess whether miR-744 directly targeted PTEN, a vector containing the PTEN-3'UTR was constructed and transfected into MG63 cells. It was demonstrated that the miR-744 precursor decreased luciferase activity, whereas anti-miR-744 significantly increased the luciferase activity of MG63 cells transfected with the wild-type PTEN 3'UTR (Fig. 3B). Furthermore, the protein expression of PTEN was significantly reduced by miR-744 overexpression, but significantly increased by miR-744 inhibition in osteosarcoma cells (Fig. 3C and D).

PTEN functions as a negative regulator of the PI3K/AKT signaling pathway, which has been reported to be active in a wide range of tumor types (16-18). Therefore, the present study 
A

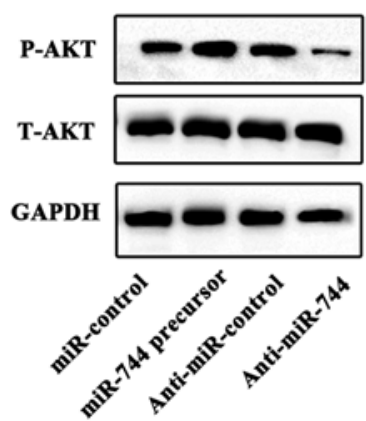

B

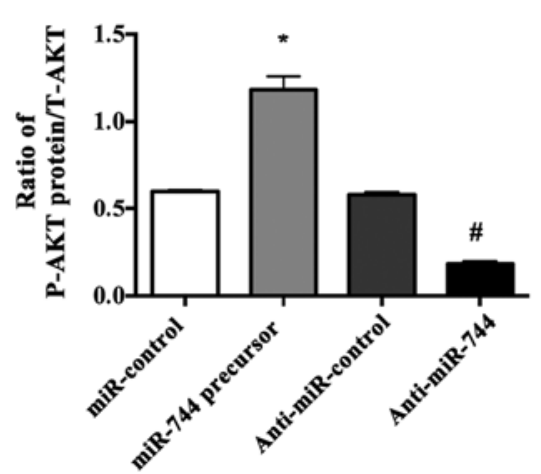

C

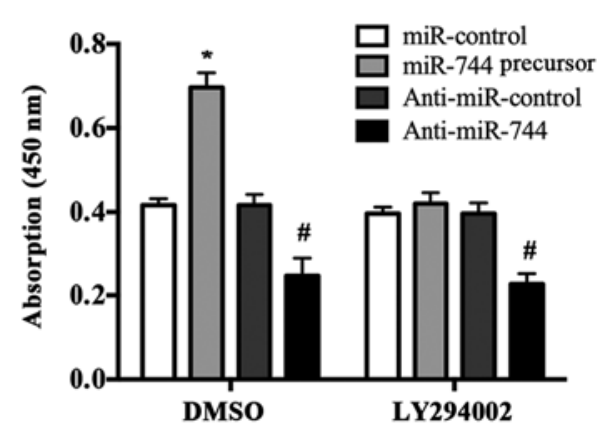

Figure 4. miR-744 positively regulates AKT activation in MG63 cells. (A) Representative images and (B) semi-quantitative analysis of the expression of P-AKT in MG63 cells was obtained by western blotting, following transfection with miR-744 precursor, anti-miR-744 and negative controls for $24 \mathrm{~h}$. GAPDH was used as a loading control. (C) Bromodeoxyuridine assay detected the proliferative potential of MG63 cells following transfection with miR-744 precursor or anti-miR-744 for $24 \mathrm{~h}$. Cells were pretreated with DMSO or $20 \mu \mathrm{M} \mathrm{LY} 294002$ for $8 \mathrm{~h}$. GAPDH was used as an internal control. "P<0.05 vs. miR-control; ${ }^{\text {"P}} \mathrm{P}<0.05$ vs. Anti-miR-control. miR, microRNA; P-, phosphorylated; T-, total.

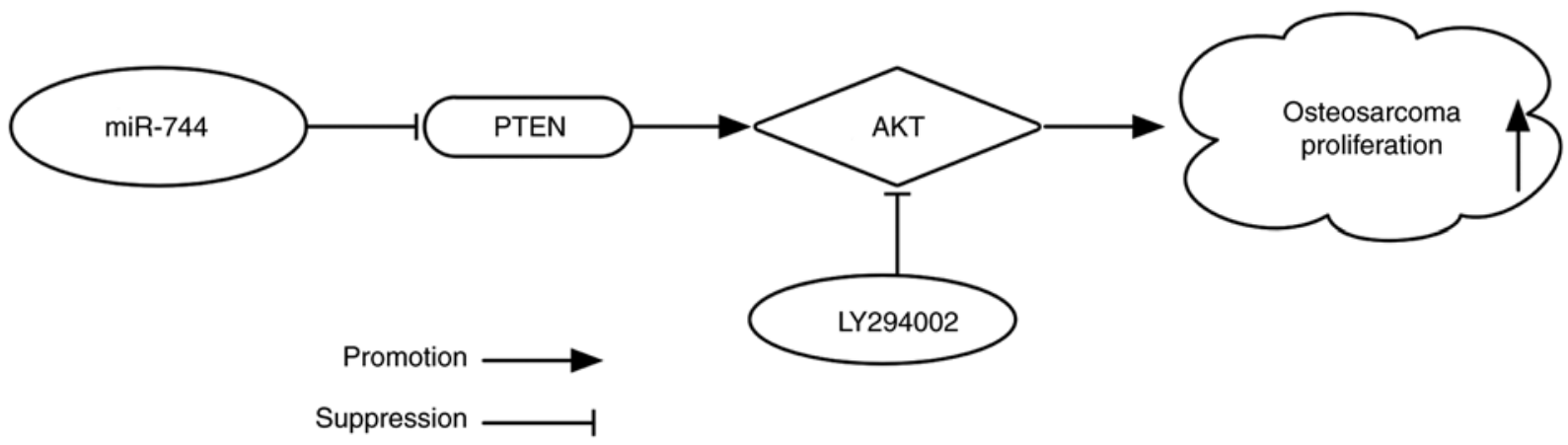

Figure 5. Schematic diagram of the mechanism of action of miR-744 in osteosarcoma cell proliferation. miR, microRNA.

detected the protein expression of active AKT via western blotting in osteosarcoma cells transfected with miR-744 precursor or anti-miR-744. It was identified that miR-744 overexpression induced AKT activation in MG63 cells, whereas downregulation of miR-744 decreased P-AKT expression (Fig. 4A and B). In addition, LY294002, an AKT inhibitor, abrogated the proliferative effect induced by miR-744 overexpression in osteosarcoma cells (Fig. 4C). Collectively, the present results indicated that PTEN may be a direct target of miR-744 in osteosarcoma cells.

\section{Discussion}

Osteosarcoma, one of the most malignant types of bone cancer, is associated with rapid deterioration and metastasis, and is a major health threat to pediatric and adolescent patients $(19,20)$. Previous studies have shown that miRNAs are involved in the regulation of various biological processes in osteosarcoma, including cell proliferation, differentiation, migration and invasion (7-9). The present results suggested that miR-744 expression was upregulated in osteosarcoma tissues and cells. Moreover, it was demonstrated that ectopic overexpression of miR-744 promoted the proliferation of MG63 cells, whereas miR-744 inhibition using antisense oligonucleotides inhibited cell proliferation.
It has been reported that miR-744 may function as either an oncogene or a tumor suppressor in different cancer types. Consistent with the present results, miR-744 was highly expressed and acted as an oncogene in pancreatic cancer (21), prostate cancer (22) and nasopharyngeal carcinoma (23). Furthermore, overexpression of miR-744 promoted tumor metastasis by suppressing PTEN and programmed cell death protein 4 pathways in laryngeal squamous cell carcinoma (12). However, other studies have suggested a role for miR-744 as a tumor suppressor rather than a dominant oncogene. miR-744 was downregulated and acted as a tumor suppressor in glioblastoma (24), gastric cancer (25) and colorectal cancer (26). Moreover, low expression of miR-744 has been implicated in the development and progression of liver cancer, and thus may be considered a potential prognostic marker in patients with hepatocellular carcinoma (27). Therefore, it is speculated that the dual roles of miR-744 may be attributed to the different cellular contexts and its interaction with target genes. Sun et al (11) revealed that miR-744 promoted osteosarcoma carcinogenesis by inhibiting the LATS2 signaling pathway. Furthermore, Sun et al (11) conducted cell viability, migration and invasion assays, and reported that miR-744 promoted cell viability and metastasis of osteosarcoma by suppressing LATS2 signaling. However, the biological functions and the underlying molecular mechanisms of miR-744 
in osteosarcoma are not fully understood. Therefore, the present study investigated whether the oncogene miR-744 promoted osteosarcoma via a novel PTEN signaling pathway. The present study revealed that miR-744 was overexpressed in osteosarcoma tissues and cell lines, and also demonstrated that miR-744 promoted osteosarcoma cell proliferation via the suppression of PTEN expression. Thus, the present results may increase the understanding of the functional role of miR-744 in osteosarcoma, as it was indicated that miR-744 may primarily act as an oncogenic miRNA in osteosarcoma. However, whether miR-744 promotes cell migration and invasion of osteosarcoma via the PTEN pathway in vivo and in vitro requires further investigation.

PTEN is a tumor suppressor gene, and loss of its expression has been reported to be associated with growth and metastasis of various malignant tumors (13). PTEN deficiency may also result in activation of the PI3K/AKT signaling pathway, thus inhibiting apoptosis and promoting proliferation of tumor cells $(16,28)$. Previous studies showed that the PTEN/PI3K/AKT signaling pathway was critical for carcinogenesis and progression in numerous cancer types, and AKT phosphorylation has been used as an indicator of activation of this pathway (29-31). Moreover, miR-744, acting as an oncogenic miRNA, has been reported to promote metastasis of laryngeal squamous cell carcinoma by suppressing PTEN expression and activating the PI3K/AKT signaling pathway (12). The present results indicated that miR-744 promoted cell proliferation in MG63 cells, which was associated with reduced PTEN expression and increased AKT phosphorylation. In addition, it was demonstrated that blocking the PI3K/AKT pathway with LY294002, an AKT inhibitor, abrogated the proliferative effect induced by $\mathrm{miR}-744$. Therefore, the present results suggested that miR-744 increased osteosarcoma cell proliferation via the suppression of PTEN and subsequent activation of the PI3K/AKT pathway.

In conclusion, the present study demonstrated that miR-744 was overexpressed in osteosarcoma tissues and cells. miR-744 was suggested to have an active role in promoting cell proliferation via suppression of the PTEN signaling pathway (Fig. 5). These results may provide novel insights into the pathophysiological mechanism of osteosarcoma and facilitate the development of therapeutic targets for the treatment of this cancer type. However, there are some limitations to the present study. For example, this experiment was primarily conducted at the cellular level, and further in vivo studies are required to elucidate the role of miR-744 in osteosarcoma. Moreover, further experiments are required to investigate miR-744 regulation, as well as its underlying mechanisms in cell migration and invasion of osteosarcoma.

\section{Acknowledgements}

Not applicable.

\section{Funding}

The present study was supported by the Zhejiang Province Technology Project (grant no. 2015C33209) and the Wenzhou Technology Project (grant no. Y20150243).

\section{Availability of data and materials}

The datasets used and/or analyzed through the current study are available from the corresponding author upon reasonable request.

\section{Authors' contributions}

WY performed the majority of experiments and drafted the manuscript. PBC, FCC and SLD assisted with the experiments. WY and PBC analyzed the data and drafted the manuscript. WY and XYP conceived the study, supervised the experiments and edited the manuscript. All authors read and approved the final manuscript.

\section{Ethics approval and consent to participate}

The present study was approved by the Ethics Committee of The Second Affiliated Hospital, Wenzhou Medical University. Informed consent was obtained from the patients (age, $\geq 18$ years) or their parents (patients aged $<18$ years).

\section{Patient consent for publication}

Not applicable.

\section{Competing interests}

The authors declare that they have no competing interests.

\section{References}

1. Ameres SL and Zamore PD: Diversifying microRNA sequence and function. Nat Rev Mol Cell Biol 14: 475-488, 2013.

2. Ambros V: The functions of animal microRNAs. Nature 431: 350-355, 2004.

3. Esquela-Kerscher A and Slack FJ: Oncomirs-microRNAs with a role in cancer. Nat Rev Cancer 6: 259-269, 2006

4. Calin GA and Croce CM: MicroRNA signatures in human cancers. Nat Rev Cancer 6: 857-866, 2006.

5. Saraf AJ, Fenger JM and Roberts RD: Osteosarcoma: Accelerating progress makes for a hopeful future. Front Oncol 8: 4, 2018.

6. Xu J, Wang H, Hu Y, Zhang YS, Wen L, Yin F, Wang Z, Zhang Y, Li S, Miao Y, et al: Inhibition of CaMKII $\alpha$ activity enhances antitumor effect of fullerene C60 nanocrystals by suppression of autophagic degradation. Adv Sci (Weinh) 6: 1801233-1801233, 2019.

7. Liu JL, Li J, Xu JJ, Xiao F, Cui PL, Qiao ZG, Chen XD, Tao WD and Zhang XL: miR-144 inhibits tumor growth and metastasis in osteosarcoma via dual-suppressing RhoA/ROCK1 signaling pathway. Mol Pharmacol 95: 451-461, 2019.

8. Jianwei Z, Fan L, Xiancheng L, Enzhong B, Shuai L and Can L: MicroRNA 181a improves proliferation and invasion, suppresses apoptosis of osteosarcoma cell. Tumour Biol 34: 3331-3337, 2013.

9. Chen Z, Zhao G, Zhang Y, Ma Y, Ding Y and Xu N: miR-199b-5p promotes malignant progression of osteosarcoma by regulating HER2. J BUON 23: 1816-1824, 2018.

10. Zhu KP, Zhang CL, Ma XL, Hu JP, Cai T and Zhang L: Analyzing the interactions of mRNAs and ncRNAs to predict competing endogenous RNA networks in osteosarcoma chemo-resistance. Mol Ther 27: 518-530, 2019.

11. Sun L, Liu M, Luan S, Shi Y and Wang Q: MicroRNA-744 promotes carcinogenesis in osteosarcoma through targeting LATS2. Oncol Lett 18: 2523-2529, 2019.

12. Li JZ, Gao W, Lei WB, Zhao J, Chan JY, Wei WI, Ho WK and Wong TS: MicroRNA 744-3p promotes MMP-9-mediated metastasis by simultaneously suppressing PDCD4 and PTEN in laryngeal squamous cell carcinoma. Oncotarget 7: 58218-58233, 2016. 
13. Tamura M, Gu J, Matsumoto $\mathrm{K}$, Aota S, Parsons $\mathrm{R}$ and Yamada KM: Inhibition of cell migration, spreading, and focal adhesions by tumor suppressor PTEN. Science 280: 1614-1617, 1998.

14. Rosenberg AE, Cleton-Jansen AM, de Pineux G, Deyrup AT, Hauben E and Squire J: Conventional osteosarcoma. In: WHO Classification of Tumours of Soft Tissue and Bone. 4th edition. Fletcher CDM, Bridge JA, Hogendoorn CWH and Mertens F (eds). IARC Press, Lyon, pp282-288, 2013.

15. Livak KJ and Schmittgen TD: Analysis of relative gene expression data using real-time quantitative PCR and the 2(-Delta Delta C(T)) method. Methods 25: 402-408, 2001.

16. Cantley LC and Neel BG: New insights into tumor suppression: PTEN suppresses tumor formation by restraining the phosphoinositide 3-kinase/AKT pathway. Proc Natl Acad Sci USA 96: 4240-4245, 1999.

17. Sansal I and Sellers WR: The biology and clinical relevance of the PTEN tumor suppressor pathway. J Clin Oncol 22: 2954-2963, 2004.

18. Yang H, Kong W, He L, Zhao JJ, O'Donnell JD, Wang J, Wenham RM, Coppola D, Kruk PA, Nicosia SV and Cheng JQ: MicroRNA expression profiling in human ovarian cancer: miR-214 induces cell survival and cisplatin resistance by targeting PTEN. Cancer Res 68: 425-433, 2008

19. Zhang Y, Wang F, Li M, Yu Z, Qi R, Ding J, Zhang Z and Chen X: Self-Stabilized hyaluronate nanogel for intracellular codelivery of doxorubicin and cisplatin to osteosarcoma. Adv Sci (Weinh) 5: 1700821,2018

20. Li S, Zhang T, Xu W, Ding J, Yin F, Xu J, Sun W, Wang H, Sun M, Cai Z and Hua Y: Sarcoma-targeting peptide-decorated polypeptide nanogel intracellularly delivers shikonin for upregulated osteosarcoma necroptosis and diminished pulmonary metastasis. Theranostics 8: 1361-1375, 2018

21. Zhou W, Li Y, Gou S, Xiong J, Wu H, Wang C, Yan H and Liu T: miR-744 increases tumorigenicity of pancreatic cancer by activating Wnt/ $\beta$-catenin pathway. Oncotarget 6: 37557-37569, 2015 .
22. Zhang M, Li H, Zhang Y and Li H: Oncogenic miR-744 promotes prostate cancer growth through direct targeting of LKB1. Oncol Lett 17: 2257-2265, 2019.

23. Yu Q, Zhang F, Du Z and Xiang Y: Up-regulation of serum miR-744 predicts poor prognosis in patients with nasopharyngeal carcinoma. Int J Clin Exp Med 8: 13296-13302, 2015.

24. Deng Y, Li Y, Fang Q, Luo H and Zhu G: microRNA-744 is downregulated in glioblastoma and inhibits the aggressive behaviors by directly targeting NOB1. Am J Cancer Res 8: 2238-2253, 2018

25. Xu AJ, Fu LN, Wu HX, Yao XL and Meng R: MicroRNA744 inhibits tumor cell proliferation and invasion of gastric cancer via targeting brainderived neurotrophic factor. Mol Med Rep 16: 5055-5061, 2017.

26. Shen J and Li M: MicroRNA-744 inhibits cellular proliferation and invasion of colorectal cancer by directly targeting oncogene Notch1. Oncol Res Feb 22, 2018 (Epub ahead of print).

27. Tan YL, Bai ZG, Zou WL, Ma XM, Wang TT, Guo W, Liu J, Li JS, Jie-Yin, Zang YJ and Zhang ZT: miR-744 is a potential prognostic marker in patients with hepatocellular carcinoma. Clin Res Hepatol Gastroenterol 39: 359-365, 2015.

28. Osaki M, Oshimura M and Ito H: PI3K-Akt pathway: Its functions and alterations in human cancer. Apoptosis 9: 667-676, 2004.

29. Colakoglu T, Yildirim S, Kayaselcuk F, Nursal TZ, Ezer A Noyan T, Karakayali H and Haberal M: Clinicopathological significance of PTEN loss and the phosphoinositide 3-kinase/Akt pathway in sporadic colorectal neoplasms: Is PTEN loss predictor of local recurrence? Am J Surg 195: 719-725, 2008.

30. Saal LH, Holm K, Maurer M, Memeo L, Su T, Wang X, Yu JS, Malmström PO, Mansukhani M, Enoksson J, et al: PIK3CA mutations correlate with hormone receptors, node metastasis, and ERBB2, and are mutually exclusive with PTEN loss in human breast carcinoma. Cancer Res 65: 2554-2559, 2005.

31. Tang JM, He QY, Guo RX and Chang XJ: Phosphorylated Akt overexpression and loss of PTEN expression in non-small cell lung cancer confers poor prognosis. Lung Cancer 51: 181-191, 2006. 